Prix Nobel de Physique 2018

Arthur Ashkin, Gérard Mourou et Donna Strickland

\section{Trois chercheurs, dont un Français, récompensés pour leurs travaux sur les lasers}

Serge R. Mordon

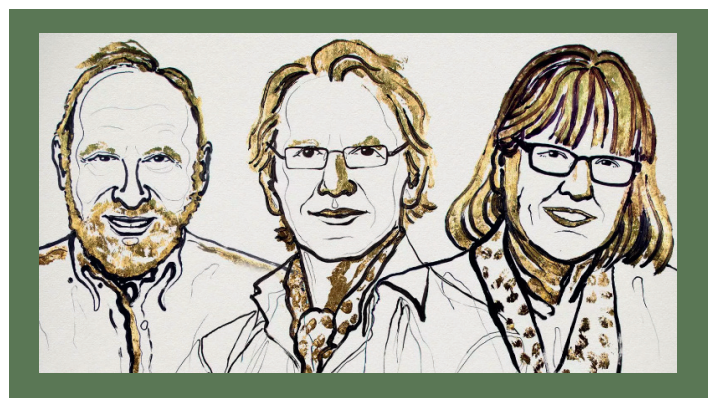

OncoThAl, Inserm U1189, Université de Lille, CHU de Lille, 1, avenue 0 scar Lambret, 59000 Lille, France.

serge.mordon@inserm.fr

puissance peut atteindre le pétawatt $\left(10^{15} \mathrm{~W}\right)$. Pour fixer les ordres de grandeur, si, en une seconde, la lumière parcourt une distance équivalente à 7 fois le tour de la Terre, en une femtoseconde, elle n'a pas le temps de traverser l'épaisseur d'un cheveu, et... à peine celle d'un virus. La puissance solaire reçue par la Terre est, quant à elle, de 175 pétawatts.

Les applications de la technique CPA sont très nombreuses: de la fusion contrôlée par confinement inertiel jusqu'à la biophysique en passant par la spectroscopie moléculaire ultrarapide. La plupart des laboratoires académiques comme industriels possèdent en général un laser à impulsions femtosecondes. Ces impulsions sont utilisées pour forer ou trancher de nombreux matériaux. En médecine, elles permettent d'inciser avec une précision inégalée les tissus biologiques sans aucun dommage pour les structures tissulaires adjacentes. Aujourd'hui, l'ophtalmologie a recours quotidiennement à ces lasers femtosecondes pour la chirurgie réfractive. L'article de Christophe Baudouin, du Centre National d'Ophtalmologie des Quinze-Vingts et de l'institut de la Vision à Paris nous explique en détails les bénéfices apportés par cette évolution technologique dans son domaine [1] $(\rightarrow)$.

$(\rightarrow)$ Voir l'article Nobel de C. Baudouin, page 178 de ce numéro La coupe de l'os requise lors de nombreuses interventions chirurgicales pourrait devenir aussi très bientôt une application très utile de ces lasers [2]. On pourrait aussi en citer de très nombreuses autres, telles que la microscopie à deux photons, une technique d'imagerie par 
microscopie à fluorescence qui permet une image d'un tissu vivant à une profondeur d'un millimètre.

L'interaction d'une impulsion laser femtoseconde avec une cible solide peut aussi induire une émission intense de rayonnement $X$ dans la gamme de quelques dizaines de kiloélectronvolts (keV, soit mille électronvolts). Ce type de source présente potentiellement plusieurs avantages sur les installations $X$ conventionnelles en termes de performances et de coût. À Bordeaux par exemple, le projet Xpulse vise à développer un système innovant d'imagerie médicale par rayons $X$ obtenus au moyen d'impulsions femtosecondes pour une application dans la détection précoce du cancer du sein. La résolution de ces systèmes, supérieure à la radiographie standard, devrait permettre de détecter des cancers du sein de petite taille à des stades plus précoces ${ }^{1}$.

\section{Arthur Ashkin}

L'Américain Arthur Ashkin, chercheur à la retraite des laboratoires Bell (aux États-Unis), a quant à lui été récompensé pour ses travaux novateurs en optique non-linéaire ayant mené à l'invention des pinces optiques (optical tweezers, en anglais). Elles sont utilisées pour la micromanipulation d'atomes dans les condensats de type Bose-Einstein ${ }^{2}$ ou d'éléments biologiques, tels que des cellules vivantes, mais aussi des virus ou des protéines. Arthur Ashkin a en effet réussi à démontrer qu'un laser pouvait exercer une force suffisante (pression de radiation) sur une sphère pour la mettre en mouvement ou la faire se déplacer, comme si on la tenait à l'aide d'une petite pince. Les forces générées par les pinces optiques sont typiquement équivalentes aux forces mises en jeu dans un grand nombre de processus cellulaires (adhérence, mécanique du cytosquelette, motricité, fonctionnement des moteurs moléculaires, etc.) [3].

${ }^{1}$ https://www.photoniques.com/actualites/rad/3861-alphanov-au-coeur-du-projet-xpulse

${ }^{2}$ La condensation de Bose-Einstein a été imaginée par Albert Einstein et le physicien indien Satyendranath Bose dès 1924. En refroidissant un nuage d'atomes à une température proche du zéro absolu, un tel condensat, état de la matière qui n'est ni solide ni liquide mais ressemblerait plutôt à un gaz pris en masse, a été produit.
En 1987, Ashkin a réussi à capturer avec cette technique des bactéries vivantes sans les endommager et en conservant un milieu stérile. Ce nouvel outil est maintenant utilisé quotidiennement par de très nombreux chercheurs, notamment en biologie, car ces pinces s'intègrent facilement dans des systèmes d'imagerie microscopique auxquels elles ajoutent la capacité de manipuler et de contrôler à l'échelle nanométrique les objets observés. Il a ainsi été possible de séparer les brins de la double hélice de l'ADN grâce à l'action d'une force mécanique exercée avec une pince optique. On a pu ainsi déterminer la force avec laquelle sont liées les paires de bases azotées et établir que ces forces variaient selon la séquence de paires de bases.

Un autre grand domaine d'applications concerne l'étude et la fabrication de nano- ou de micromoteurs pour la micro-fluidique. Cette technique, combinée à l'utilisation des pinces optiques, est à la base des micro-laboratoires (lab-on-a-chip), des laboratoires utilisant des microtechniques, miniaturisant les méthodes des laboratoires conventionnels. $\nabla$

Three researchers, including a Frenchman, rewarded for their work on lasers

\section{LIENS D'INTÉRÊT}

L'auteur déclare n'avoir aucun lien d'intérêt concernant les données publiées dans cet article.

\section{RÉFÉRENCES}

1. Baudouin C. Arthur Ashkin, Gérard Mourou, Donna Strickland, Prix Nobel de physique 2018 : un Nobel, des applications. Med Sci (Paris) 2019 ; 35 : 178-80.

2. Gill RK, Smith ZJ, Lee C, Wachsmann-Hogiu S. The effects of laser repetition rate on femtosecond laser ablation of dry bone: a thermal and LIBS study. J Biophotonics 2016; 9 : 171-80.

3. Galaup JP. La pince optique. Photoniques $2013 ; 66: 51-5$.

TIRÉs À PART

S.R. Mordon

\section{AMPS (Association Médecine Pharmacie Sciences)}

La période de candidature pour la passerelle permettant d'intégrer directement les études de Santé (Médecine, Pharmacie, Odontologie et Maïeutique) après un diplôme de niveau Bac +5 ou plus approche à grand pas. Pour cette occasion, l'AMPS (Association Médecine Pharmacie Sciences) a le plaisir de vous présenter son guide de candidature au concours passerelle, ainsi que son guide pour préparer sa rentrée directement en $2^{\mathrm{e}}$ ou $3^{\mathrm{e}}$ année des études de Santé, rédigé par des étudiants qui s'y trouvent déjà

Si vous êtes un candidat potentiel, ou que vous en connaissez, n’hésitez pas à les utiliser, ils contiennent théoriquement toutes les informations utiles pour passer du la candidature à la rentrée dans l'année supérieure. Et n'hésitez pas non plus à nous faire part de toutes vos remarques concernant ces guides, nous nous ferons un plaisir de les améliorer.

Enfin, si vous-même avez bénéficié de cette passerelle par le passé, n’hésitez pas à nous contacter pour nous faire part de vos témoignages, vos successeurs vous en remercieront grandement.

Vous trouverez toutes les informations intéressantes sur le site de l'AMPS: https://amps-asso.org/ > Opportunités > Guides Yanis Bendjelal, rédacteur en chef des guides Sciences-Santé de l'AMPS 